prevalence of atherosclerosis. This has been characteristically attributed to the inflammation present in both diseases

Objectives: To analyze the differences in the role of traditional cardiovascular risk factors in the subclinical atherosclerosis between the two diseases.

Methods: Cross-sectional study encompassing 602 subjects, 276 SLE and 326 RA patients. Carotid plaques were assessed by ultrasonography. A multivariable regression analysis was performed to evaluate if classic cardiovascular-related risk factors influence differentially on subclinical carotid atherosclerosis in SLE compared to RA patients.

Results: Traditional cardiovascular risk factors were found to be prevalent in RA and SLE patients. Age (interaction factor $p=0.000$ ), hypertension (interaction factor $\mathrm{p}=0.034$ ), and diabetes (interaction factor $\mathrm{p}=0.037$ ) were found to have a higher effect on CIMT in RA patients compared to SLE subjects. No differences between RA and SLE were discovered in the effect of traditional cardiovascular factors over the presence of carotid plaque when univariate interaction was performed. After final adjustment for demographics, the presence of others traditional cardiovascular factors, and disease related data, no differences were found in the influence of hypertension, diabetes, dyslipidemia or current smoking over cIMT or the presence of carotid plaque. Moreover, the effect of the addition of various cardiovascular risk factors on the subclinical carotid atherosclerosis did not differ between both diseases.

Conclusion: The influence of traditional cardiovascular risk factors (hypertension, diabetes, dyslipemia and smoking) over cIMT and carotid plaque is equal in RA and SLE. No interaction was found between traditional cardiovascular risk factors and disease related data in the effect of the former on subclinical atherosclerosis.

Disclosure of Interests: Hiurma Sánchez-Pérez: None declared, Juan Carlos Quevedo-Abeledo: None declared, Iñigo Rúa-Figueroa: None declared, Beatriz Tejera-Segura: None declared, Vanessa Hernández-Hernández: None declared, Antonia de Vera-González: None declared, Alejandra Delgado-González: None declared, Raquel López-Mejías: None declared, Soledad Ojeda Grant/research support from: AMGEN, Speakers bureau: AMGEN, Miguel A González-Gay Grant/research support from: Prof. MA Gonzalez-Gay received grants/research supports from Abbvie, MSD, Jansen and Roche., Speakers bureau: Consultation fees/participation in company sponsored speaker's bureau from Pfizer, Lilly, Sobi, Celgene, Novartis, Roche and Sanofi., Iván Ferraz-Amaro: None declared DOI: 10.1136/annrheumdis-2019-eular.1845

\section{FRI0251 SYSTEMATIC REVIEW OF PREDICTOR MARKERS OF POOR RENAL EVOLUTION IN PATIENTS WITH LUPUS NEPHRITIS}

Esther Rodríguez Almaraz ${ }^{1}$, Elena Gutiérrez Solís ${ }^{2}$, Elena Rabadan ${ }^{1}$, Paola Rodríguez Ramos ${ }^{2}$, Enrique Morales Ruiz², María Galindo-Izquierdo'. ${ }^{1}$ Hospital 12 De Octubre, Rheumatology, Madrid, Spain; ${ }^{2}$ Hospital 12 De Octubre, Nephrology, Madrid, Spain

Background: Lupus nephritis (LN) affects $30-45 \%$ of patients with lupus and causes great morbidity and mortality. About $10-25 \%$ of patients will develop chronic kidney disease (CKD), and it has been described a mortality of $10-20 \%$ at 10 years $_{1-2}$. Currently there is no ideal biomarker or composite indexes that help us to better assess and monitor.

Objectives: To identify and perform a critical analysis of the prognostic markers and indexes published through a systematic review of the literature in order to subsequently develop an index that helps us to estimate the prognosis of patients with LN

Methods: We conducted a systematic review and meta-analysis according to the guidelines of the "Cochrane Collaboration" and our results are exposed in accordance with the "Preferred Reporting Items for Systematic Reviews and Meta-Analyzes PRISMA statement". We did an electronic search in MEDLINE, EMBASE, CINAHL and Web of Science using free text and MeSH terms. The selection was made by 2 independent authors using a bibliographic manager. First, a selection was made by title and if it seemed relevant, a review of the abstract was made. In case of doubt, the full text was evaluated for a possible inclusion. The articles had to fulfill the following criteria: marker or prognostic index of LN, longitudinal studies, to collect the prognostic factor retrospectively without variable biases suitable for good/poor renal prognosis and LN diagnosed by biopsy. Six authors independently extracted data from the articles on previously tested forms to determine their feasibility, and differences were discussed. The data included the general characteristics and results measured. Also adverse events were reported.

Results: We selected 1010 articles initially, of which we discarded 853 per title. Of the 157 remaining, 84 were selected per abstract, and finally, 62 articles were excluded after the review of the full text. In total, the 22 studies included 2301 patients, all older than 18 years. Most were single-center and retrospective (65\%). Among the analytical markers, serum creatinine $(\mathrm{Cr})$ was one of the most used variables as a predicto of time to CKD depending on the value, which varied according to the studies. It was also associated as a damage predictor according to the SLICC scale. Proteinuria appears in several studies as a marker of progression of $\mathrm{LN}$, associating the amount with renal response, the develop ment of end-stage renal disease (ESRD), the need for dialysis and the appearance of extrarenal events. Other variables analyzed were: demographic (ethnicity, socioeconomic level), immunological (anti-Ro, anti-DNA anti-C1q and anti-CRP antibodies with poor renal evolution), histopathological (increased nedd for dialysis in glomerulonephritis IV, thrombotic microangiopathy, C4d deposits or tubular atrophy and association of chronicity index with remission) or therapeutic (hydroxychloroquine with remission). Conclusion: Altough there are several biomarkers to evaluate the LN prognosis, we have no found indexes for LN prognosis assessment in literature. The development of easily reproducible compound index is necessary for the assessment of the prognosis and the severity of patients with LN on debut and during evolution.

\section{REFERENCES}

[1] Carmona L, Gabriel R, Ballina J, Laffon A. Proyecto EPISER 2000: prevalencia de enfermedades reumáticas en la población española. Metodología, resultados del reclutamiento y características de la población. $2001 ; 28: 8$.

[2] Austin HA, Boumpas DT, Vaughan EM, Balow JE. High-risk features of lupus nephritis: importance of race and clinical and histological factors in 166 patients. Nephrol Dial Transplant Off Publ Eur Dial Transpl Assoc Eur Ren Assoc. 1995;10(9):1620-8.

Disclosure of Interests: None declared

DOI: 10.1136/annrheumdis-2019-eular.6222

\section{FRI0252 CLINICAL AND SEROLOGICAL LUPUS ACTIVITY BEFORE AND AFTER DEVELOPING END STAGE RENAL DISEASE}

Maria Salgado Guerrero ${ }^{1}$, Alejandra Londono Jimenez ${ }^{2}$, Chrisanna Dobrowolsky ${ }^{2}$, Shudan Wang ${ }^{2}$, Anna R. Broder'. ' Jacobi Medical Center/Albert Einstein College of Medicine, Internal Medicine, Bronx, NY, United States of America: ${ }^{2}$ Montefiore Medical Center/Albert Einstein College of Medicine, Rheumatology, Bronx, NY, United States of America

Background: SLE disease activity tends to diminish after the development of end stage renal disease $(E S R D)^{1,2}$. Nonetheless, some patients con tinue to show signs of active disease and experience flares after ESRD ${ }^{3}$. To date, little is known about the evolution of SLE-related symptoms pre and post ESRD. Whether specific symptoms abate after ESRD or a shift towards different manifestations occurs deserves further study. Prompt identification of subtler SLE manifestations is further complicated by a trend towards poor rheumatology follow once ESRD develops. SLE manifestations post-ESRD may be underdiagnosed and undertreated contribu ting to increased morbidity and mortality.

Objectives: To study the different clinical manifestations and serological markers of SLE disease activity before and after ESRD development. Methods: We performed a retrospective chart review of SLE patients with ESRD at a tertiary care center between the years of 2010 and 2017 SLE was defined by ACR and/or SLICC criteria. SLE ESRD patients were included if they had at least one visit with rheumatology, nephrology, or primary care pre- and post-ESRD. SLE-related symptoms and serologic markers of disease activity were identified from chart review before and after ESRD onset.

Results: Fifty-eight patients were included. Twenty-five patients had a least one clinical non-renal criteria documented pre-ESRD. Of them, 14 achieved complete clinical remission post-ESRD. Post-ESRD, cytopenias persisted in 49 of the 55 patients who were cytopenic pre-ESRD. Arthritis persisted in 3 of the 13 patients who had arthritis pre-ESRD. Of the 37 patients with hypocomplementemia pre-ESRD, 29 remained hypocomplementemic post-ESRD. Twenty-nine had elevated dsDNA pre-ESRD, of them, dsDNA remained elevated in 16 patients post-ESRD. Six patients developed at least one new clinical criteria post-ESRD. Three patients developed low complement, 5 developed elevated dsDNA, 3 developed new onset of serositis and 4 new osent of arthritis post-ESRD.

Conclusion: Lupus activity may diminish after ESRD onset. However, many patients experience persistent disease activity. New arthritis, serosi tis, low complements and elevated dsDNA may develop after ESRD. Limited evaluation and documentation of disease activity by non-rheumatology 